\title{
Editorial for "Diffusion Tensor Imaging for Quantitative Assessment of Anterior Cruciate Ligament Injury Grades and Graft"
}

\author{
Dimitri A Kessler, MS, ${ }^{1 \dagger}$, James W MacKay, PhD, ${ }^{1,2}$, Stephen McDonnell, FRCS, ${ }^{3}$ and \\ Joshua D Kaggie, $\mathrm{PhD}^{1}$ \\ ${ }^{1}$ Department of Radiology, University of Cambridge, United Kingdom. \\ ${ }^{2}$ Norwich Medical School, University of East Anglia, Norwich, United Kingdom \\ ${ }^{3}$ Division of Trauma and Orthopaedics, University of Cambridge, Cambridge, United \\ Kingdom
}

Tears to the anterior cruciate ligament (ACL) are common and serious knee injuries which tend to occur in young, active individuals. They result in functional impairment and require a period of relative immobilisation followed by rehabilitation, often leading to surgery. Individuals suffering from an ACL injury also have a higher risk of developing osteoarthritis as a longterm consequence $(1,2)$. ACL reconstructive surgery using a tendon graft remains the clinical standard of care to provide stability to the knee joint and allow patients to return to sport quicker. However, the question of when to allow patients to return to high-level sport remains hotly debated, as the risk of sustaining a second ACL rupture following reconstructive surgery is highest within the subsequent two years(3). While conventional MRI methods continue to provide high diagnostic structural information for ACL injuries, they are unable to deliver advanced quantitative measures required for biological tissue characterisation and longitudinal observation of graft maturation. Promising techniques such as diffusion tensor imaging (DTI), are used for research purposes only and have not yet made the translation into routine clinical application.

The study "Diffusion Tensor Imaging for Quantitative Assessment of Anterior Cruciate Ligament Injury Grades and Graft" by ... et al(4) published in this issue of the Journal of Magnetic Resonance Imaging evaluates two promising clinical applications of DTI. Firstly, 
DTI-derived measures are shown for the diagnostic assessment of anterior cruciate ligament (ACL) injury grades, and secondly, the longitudinal assessment of ACL grafts in patients having undergone reconstructive surgery is shown with DTI.

Thirty-five patients across four different arthroscopically confirmed ACL injury grades (I-IV) and twenty healthy controls were recruited in this study. All participants had a baseline DTI (b $=400 \mathrm{~s} / \mathrm{mm}^{2}, 20$ diffusion directions) at $3 \mathrm{~T}$ to evaluate the association between the ACL injury grade and two DTI-derived measures (fractional anisotropy (FA) and apparent diffusion coefficient (ADC)). Furthermore, 23 patients with arthroscopically confirmed ACL injury grades III and IV underwent ACL reconstructive surgery with a hamstring tendon graft; the ADCs and FAs of the inserted grafts were additionally assessed at 3-months and 6-months post-operatively.

While there have been previous studies investigating the utility of DTI for quantitative evaluation of the ACL, these were not performed acutely after ACL injury but primarily in patients that have previously undergone ACL reconstruction and in healthy control volunteers $(5,6)$. This study performed DTI in patients with four different arthroscopically confirmed ACL injury grades (I-IV) and healthy controls (0). Significant differences in FA and ADC values between all ACL injury grades (0-IV) were determined, except for the FA and ADC values between ACL injury grades I and II and the ADC values between grades III and IV. These results showed a significant negative correlation of the FA values $(r=-0.898, P<$ 0.001) with ACL injury grades, and the ADC values showed a significant positive correlation $(\mathrm{r}=+0.851, \mathrm{P}<0.001)$ with increasing grade.

Significant differences in the ADC and FA values from the grafts at 3 months post-surgery and those from the ACLs of healthy controls were determined. The ADCs and FAs at 6-months post-surgery were comparable with the values from healthy control ACLs, suggesting 
maturation of the inserted graft towards ACL-like tissue characteristics. The first study to investigate the utility of DTI for imaging the ACL graft was described by Yang et al.(5), in which 40 healthy volunteers and 15 patients, 5 months to 10 years after ACL reconstructive surgery, were imaged. Yang et al. showed that the ACL grafts displayed different FA and ADC values depending on their maturation stage with the patient having the longest period (10 years) between surgery and DTI presenting a significantly higher FA value than the other patients. While the determined FA and ADC values of healthy controls from Yang et al. were comparable to those determined in this study, those of the ACL grafts were not comparable. This difference could be explained by the more constrained recruitment strategy applied in this study and the shorter time interval between surgery and DTI. Compared to the FA values estimated in this study, Van Dyck et al.(6) determined much lower FA values 3 to 7 months post-operatively in ACL grafts imaged with DTI. Although the maturation stage of the ACL grafts should be similar, the differences could be a consequence of the variation in MRI hardware and post-processing methods.

The longitudinal analysis performed in this study is a valuable contribution towards understanding the biological changes and developments that the ACL graft experiences over 6-months post-operatively. Although we can speculate that ACL grafts appear to be undergoing "ligamentisation" processes towards a structure similar to the native ACL, as reflected by DTIderived measures, this hypothesis requires further tissue analysis. Imaging together with tissue biopsy could allow for better characterisation of compositional changes occurring during graft maturity and be used to validate the imaging results. To further improve these results, additional DTI follow-up measurements at 12- and 24-months may be helpful. A systematic review by Pauzenberger et al.(7) reported that the ACL hamstring tendon grafts mature towards native ACL-like structure after 12-24 months. Roewer et al.(8) showed that 6-months after ACL reconstructive surgery and physical therapy, knee angle, moments and power profiles as 
well as hip power profiles were asymmetric between limbs, as observed acutely after ACL injury. At 24-months post-operatively, most asymmetries of motion, strength and function were resolved. DTI could support the research and understanding on optimal graft choice for ACL reconstruction by investigating the diverse healing and maturation processes of different graft types such as quadriceps tendon, bone-patellar tendon-bone and hamstring tendon autograft.

This study encourages further research in studying and understanding the maturation process of ACL grafts as well as designing more effective clinical trials to support the translation of promising quantitative MR imaging methods, such as DTI, for longitudinal clinical investigations.

\section{References}

1. Maletius W, Messner K: Eighteen- to twenty-four-year follow-up after complete rupture of the anterior cruciate ligament. Am J Sports Med 1999; 27:711-717.

2. Lohmander LS, Östenberg A, Englund M, Roos H: High prevalence of knee osteoarthritis, pain, and functional limitations in female soccer players twelve years after anterior cruciate ligament injury. Arthritis Rheum 2004; 50:3145-3152.

3. Nagelli C V, Hewett TE: Should return to sport be delayed until two years after anterior cruciate ligament reconstruction? Biological and functional considerations. Sport Med 2017; 47:221-232.

4. Author A: Diffusion Tensor Imaging for Quantitative Assessment of Anterior Cruciate Ligament Injury Grades and Graft. J Magn Reson Imaging 2020.

5. Yang X, Li M, Chen D, et al.: Diffusion tensor imaging for anatomical and quantitative evaluation of the anterior cruciate ligament and ACL grafts: A preliminary study. J Comput 
Assist Tomogr 2014; 38:489-494.

6. Van Dyck P, Froeling M, De Smet E, et al.: Diffusion tensor imaging of the anterior cruciate ligament graft. J Magn Reson Imaging 2017; 46:1423-1432.

7. Pauzenberger L, Syré S, Schurz M: “Ligamentization” in hamstring tendon grafts after anterior cruciate ligament reconstruction: A systematic review of the literature and a glimpse into the future. Arthrosc - J Arthrosc Relat Surg 2013; 29:1712-1721.

8. Roewer BD, Di Stasi SL, Snyder-Mackler L: Quadriceps strength and weight acceptance strategies continue to improve two years after anterior cruciate ligament reconstruction. $J$ Biomech 2011; 44:1948-1953. 